\title{
Repercussões nas ações de enfermagem no cenário de pandemia do covid-19
}

\author{
Effects on nursing actions in the covid-19 pandemic scenario \\ Efectos sobre las acciones de enfermería en el escenario de pandemia covid-19
}

\begin{abstract}
RESUMO
Objetivo: descrever as repercussões da pandemia do COVID-19 nas ações de enfermagem. Método: revisão integrativa de literatura estruturada pelas seis etapas do Checklist Preferred Reporting Itens for Systematic Reviews and meta-Analyses. As bases de dados consultadas foram MEDLINE, Literatura Latino-Americana e do Caribe em Ciências da Saúde, Banco de Dados em Enfermagem e Coleciona SUS. Resultados: a amostra final compreendeu cinco artigos que apresentaram sobre a necessidade de os líderes de enfermagem reavaliar a força de trabalho e utilizar das habilidades dos profissionais existentes para atender os pacientes. Foi destacado o protagonismo do enfermeiro com sua atuação eficaz e utilizado o sistema Omaha para agilizar os atendimentos. Outras repercussões foram à piora da qualidade de vida e o aumento da angústia entre profissionais de enfermagem, falta de apoio dos líderes e a necessidade de assumir funções fora da área regular de atuação. Conclusão: reestruturar serviços de saúde, oferecer melhores condições de trabalho, remuneração e qualificação continuada significa preservar os profissionais de enfermagem e oferecer à saúde pública mundial maiores chances de sobreviver com menos impactos a outras situações de pandemia.
\end{abstract}

DESCRITORES: Enfermagem; Cuidados de Enfermagem; Condições de Trabalho; Pandemias.

\section{ABSTRACT}

Objective: to describe the repercussions of the COVID-19 pandemic on nursing actions. Method: integrative literature review structured by the six steps of the Preferred Reporting Items for Systematic Reviews and meta-Analyses checklist. The databases consulted were MEDLINE, Latin American and Caribbean Literature in Health Sciences, Database in Nursing and Coleciona SUS. Results: the final sample comprised five articles that presented the need for nursing leaders to reassess the workforce and use the skills of existing professionals to assist patients. The role of nurses with their effective performance was highlighted and the Omaha system was used to streamline care. Other repercussions were the worsening of the quality of life and the increase in anxiety among nursing professionals, lack of support from leaders and the need to assume functions outside the regular area of activity. Conclusion: restructuring health services, offering better working conditions, remuneration and continued qualification means preserving nursing professionals and offering global public health greater chances of surviving with less impact on other pandemic situations. DESCRIPTORS: Nursing; Nursing care; Work conditions; Pandemics.

\section{RESUMEN}

Objetivo: describir las repercusiones de la pandemia COVID-19 en las acciones de enfermería. Método: revisión integradora de la literatura estructurada por las seis etapas de la lista de verificación de elementos de informe preferidos para revisiones sistemáticas y metaanálisis. Las bases de datos consultadas fueron MEDLINE, Literatura Latinoamericana y Caribeña en Ciencias de la Salud, Base de Datos en Enfermería y Coleciona SUS. Resultados: la muestra final estuvo compuesta por cinco artículos que presentaron la necesidad de que los líderes de enfermería reevalúen la fuerza laboral y utilicen las habilidades de los profesionales existentes para atender a los pacientes. Se destacó el papel de las enfermeras con su desempeño eficaz y se utilizó el sistema Omaha para agilizar la atención. Otras repercusiones fueron el empeoramiento de la calidad de vida y el aumento de la ansiedad entre los profesionales de enfermería, la falta de apoyo de los líderes y la necesidad de asumir funciones fuera del ámbito habitual de actividad. Conclusion: reestructurar los servicios de salud, ofrecer mejores condiciones de trabajo, remuneración y calificación continua significa preservar a los profesionales de enfermería y ofrecer a la salud pública mundial mayores posibilidades de supervivencia con menor impacto en otras situaciones pandémicas.

DESCRIPTORES: Enfermería; Atención de Enfermería; Condiciones de Trabajo; Pandemias.

RECEBIDO EM : 29/10/2021 APROVADO EM: 06/12/2021

\section{Rangel Vinícius Xavier}

Acadêmico do curso de Enfermagem na Universidade do Estado de Minas Gerais. Unidade Divinópolis. ORCID: 0000-0002-4385-6832 


\title{
artigo
}

Xavier, R. V., Borges, M. S., Severino, G. S., Silva, M. D., Cardoso, J. P., Almeida, C. S., Souza,D. A. S.

Repercussões nas ações de enfermagem no cenário de pandemia do covid-19

\author{
Mírian de Sousa Borges \\ Acadêmica do curso de Enfermagem na Universidade do Estado de Minas Gerais. Unidade Divinópolis. \\ ORCID: 0000-0001-8452-4239
}

\section{Gabriele de Souza Severino}

Acadêmica do curso de Enfermagem na Universidade do Estado de Minas Gerais. Unidade Divinópolis. ORCID: 0000-0002-2501-9774

\section{Marina Dias da Silva}

Acadêmica do curso de Enfermagem na Universidade do Estado de Minas Gerais. Unidade Divinópolis. ORCID: 0000-0003-0820-8124

\author{
Juliana Pereira Cardoso \\ Bióloga Mestre em Bioengenharia pela Universidade Federal de São João del Rei. \\ ORCID: 0000-0002-7074-7658
}

\section{Camila Souza de Almeida}

Enfermeira Mestre em Ciências. Doutora pela Escola de Enfermagem da UFMG. Docente da Universidade do Estado de Minas Gerais. Unidade Divinópolis.

ORCID: 0000-0002-7032-0945

\section{Débora Aparecida Silva Souza}

Enfermeira Mestre em Educação em Saúde e Enfermagem. Docente da Universidade do Estado de Minas Gerais. Unidade Divinópolis.

ORCID: 0000-0002-8937-584X

\section{INTRODUÇÃO}

A pandemia do novo coronavírus (COVID-19), causado pelo vírus SARS-CoV-2 trouxe mudanças significativas na prestação de cuidado nos serviços de saúde. A nível hospitalar houve a necessidade de um aumento no número de leitos, em especial na unidade de tratamento intensivo, onde os casos agudos de COVID-19 são monitorados. Na atenção primária, teve-se a necessidade de divisão dos serviços em quatro pontos: ações de vigilância em saúde nos territórios, atenção aos usuários acometidos pela COVID, suporte aos grupos vulneráveis e continuidade das ações que já eram próprias da Atenção Primária à Saúde (APS)1.

No ano escolhido para ser o ano da enfermagem, como forma de promover maior valorização da categoria, estes profissionais vivenciaram um cenário incerto e caótico de saúde determinado pelo COVID-19. Esta circunstância permitiu relembrar os princípios essenciais do cuidado iniciados por Florence Nightingale há cerca de 200 anos. Esta classe que representa cerca de
$59 \%$ de todos os profissionais de Saúde do mundo (cerca de 28 milhões) e que estiveram na linha de frente contra o Covid-19, se destacaram na pandemia, mostrando ao mundo suas habilidades e capacidades de se adaptar e reagir rapidamente e eficientemente diante de crises2-4.

O processo de trabalho da enfermagem foi destacado como fundamental durante a pandemia do novo coronavírus. $\mathrm{O}$ enfermeiro, por meio da sua análise clínica e holística oferece um cuidado singular ao paciente, envolvendo sua família e a comunidade. Deste modo, consegue diagnosticar os problemas e propor intervenções necessárias para a manutenção da qualidade de vida do paciente, segurança da comunidade, bem como a prevenção das complicações de saúde 5 .

No cenário do COVID-19, este potencial da enfermagem levanta discussões sobre a urgência de reestruturar serviços de saúde, a começar pela Atenção Primária à Saúde que é a porta de entrada do paciente ao Sistema Único de Saúde6. Isto porque, independentemente da pandemia, o mundo sempre enfrentou problemas crônicos na saúde pública que poderiam ser enfrentados de forma mais incisiva se houvesse maiores investimentos de ampliação dos sistemas públicos de saúde e capacitação continuada da enfermagem.

A partir das mudanças nos fluxos de serviços e rotinas, os enfermeiros sofreram com a alta demanda de trabalho com os pacientes acometidos pela COVID-19. Estudo destacou que embora a competência técnica, habilidade e controle emocional sobre a prática, a equipe de enfermagem sofreu desgaste físico dada a complexidade e aumento dos casos de pacientes com COVID-196. Por sua vez, foi preciso superar os impactos negativos, mesmo sem apoio dos próprios gestores para restabelecer forças, reorganizar os pensamentos e recuperar o emocional para enfrentar uma doença desconhecida. Essa ação colaborou para desvelar as condições de trabalho, muitas vezes precárias, o adoecimento e a honrosa atuação dos profissionais que perderam suas vidas ao enfrentar a pandemia3,7.

Este destaque foi também evidenciado em estudo documental que retratou sobre a situação da enfermagem no Brasil ao revelar 
a satisfação da população nacional com os serviços prestados pela enfermagem, considerados essenciais para o enfrentamento de crise na saúde4. Contudo, sabe-se que embora todo o reconhecimento, este ainda é insuficiente para preservar a integridade física, emocional e intelectual destes profissionais. Ressalta-se que o mundo percebeu, pela primeira vez, outras ações de enfermagem inerentes à saúde como na administração de pessoas, de materiais, dos fluxos de encaminhamentos e a tomada de decisões em âmbitos individuais e coletivos4,8.

Os profissionais da linha de frente ao combate da COVID-19 foram impactados por várias situações, que afetaram não somente a dimensão física, mas principalmente a saúde mental destes. Para Duarte et al.9, os principais acometimentos mentais foram depressão, ansiedade, insônia e angústia, fatores estes que contribuíram para o afastamento de vários profissionais de seus postos de trabalho, provocando escassez de mão-de-obra. Consequentemente emergiu o sentimento de culpa, frustração, raiva e tristeza, porém, mais que isso, provocou a sobrecarga de trabalho para os demais e déficit na eficácia dos cuidados prestados9.

É notório que a pandemia aumentou o número de pacientes, e os profissionais da linha de frente, por estarem diretamente ligados ao atendimento de casos do novo coronavírus ficaram sobrecarregados de atividades, responsabilidades e susceptíveis ao desgaste psíquico, físico e emocional. Diante do exposto, este estudo teve o objetivo de descrever as repercussões da pandemia do COVID-19 nas ações de enfermagem.

\section{MÉTODOS}

Revisão integrativa de literatura para investigar, avaliar e sintetizar resultados disponíveis de pesquisas a fim de interpretar informações e os fatores relacionados de uma determinada população10. O processo de estruturação obedeceu as etapas orientadas pelo checklist Preferred Reporting Itens for Systematic Reviewsand meta-Analyses (PRISMA)11, sendo: o estabelecimento da questão norteadora; busca dos dados; determinação de critérios e extração das informações; avaliação e interpretação dos resultados seguindo pela apresentação da revisão síntese dos dados coletados. A partir dessas etapas, o presente estudo foi guiado pela questão norteadora: Quais as repercussões a pandemia do COVID-19 trouxe para o processo de trabalho das ações de enfermagem?

A busca desta investigação ocorreu no período entre os meses de junho e setembro de 2021 e foi realizada em base de dados MEDLINE, Literatura Latino-Americana e do Caribe em Ciências da Saúde (LILACS), Banco de Dados em Enfermagem (BDENF) e Coleciona SUS. Foram utilizados Descritores em Ciências da Saúde (DeCS) nos idiomas português e inglês, combinados com o operador boleano AND: Enfermagem/Nursing, Cuidados de Enfermagem/Nursing Care, Condições de Trabalho/Working Condition,
Equipe de Enfermagem/Nursing Team, COVID-19/COVID-19, Pandemias/ Pandemics. Em seguida agrupados em quatro estratégias de buscas: i) (Enfermagem) AND (Cuidados de Enfermagem) AND (Condições de Trabalho) AND (Equipe de Enfermagem) AND (COVID-19) AND (Pandemias);ii) (Enfermagem) AND (Cuidados de Enfermagem) AND (COVID-19) AND (Pandemias); iii) (Condições de Trabalho) AND (Equipe de Enfermagem) AND (COVID-19) AND (Pandemias); iv) (Equipe de Enfermagem) AND (Cuidados de Enfermagem) AND (COVID-19) AND (Pandemias).

Os critérios de inclusão foram artigos originais, em língua portuguesa e inglesa, com textos completos, publicados entre os anos 2020 e 2021, período em que ocorreu a pandemia do novo coronavírus. Os artigos elegidos precisavam descrever sobre as repercussões das ações de enfermagem

\section{Figura 1 - Fluxograma da coleta de dados. Divinópolis, MG, Brasil, 2021}

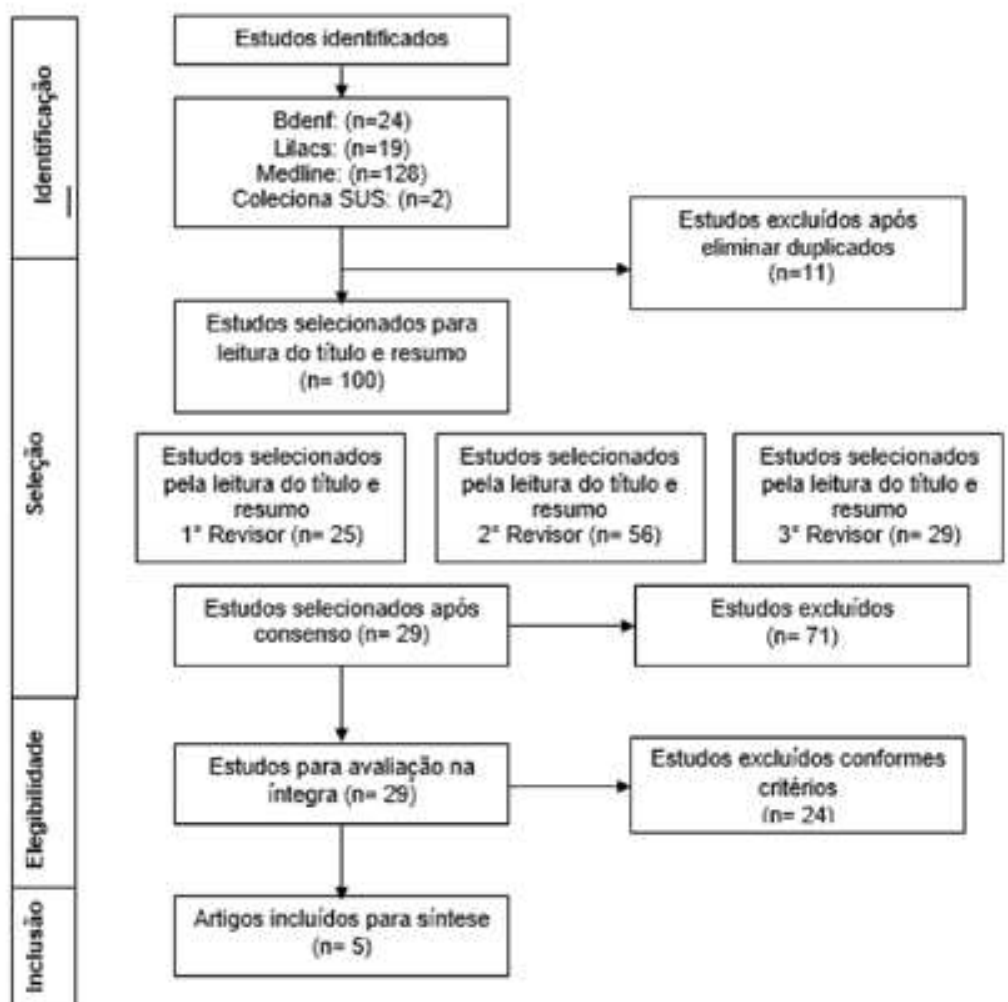

Fonte: Autores, 2021. 


\section{artigo}

Xavier, R. V., Borges, M. S., Severino, G. S., Silva, M. D., Cardoso, J. P., Almeida, C. S., Souza,D. A. S.

Repercussões nas ações de enfermagem no cenário de pandemia do covid-19

frente à pandemia de COVID-19, cenário esse que trouxe mudanças no processo de trabalho da enfermagem. Para os estudos que não estavam disponíveis na íntegra optou-se pela exclusão dada à dificuldade de acesso aos artigos fora do ambiente universitário, visto que esta revisão foi realizada em ambiente domiciliar, em período remoto de aulas ocasionado pela pandemia do SARS- CoV-2. Os critérios de exclusão foram as publicações duplicadas em bases de dados e estudos do tipo revisão e relatos de experiência.

Posteriormente, os artigos foram submetidos à avaliação pelo software Rayyan QCRL para identificar e excluir os duplicados. A etapa seguinte consistiu na leitura dos títulos, objetivos e resumos para selecionar àqueles que atenderiam aos critérios de inclusão. Três autores, de forma independente, foram responsáveis por essa etapa. Um quarto autor foi solicitado para avaliar os pontos de discordância dessa primeira triagem dos artigos.

Os artigos selecionados a partir da concordância de todos os autores e revisores foram lidos na íntegra para extração de variáveis de interesse que respondam à questão problema deste estudo, ou seja, foram excluídos aqueles que não descreviam as repercussóes da pandemia do COVID-19 para o processo de trabalho da enfermagem.

$\mathrm{Na}$ Figura 1, observa-se o fluxograma no qual foi esquematizado as etapas para alcançar a amostra final. A etapa de síntese foi desenvolvida pelo autor principal e a extração dos dados da amostra final foi organizada em uma planilha de Excel criada pelos autores com as variáveis: a) título, objetivo, delineamento metodológico, país onde o estudo foi realizado, resultados e nível de evidência dos artigos (Quadro 1).

Para conferir maior rigor de análise metodológica da avaliação dos estudos adotou-se quatro níveis de evidência sugerido pelo Centro Colaborador do Instituto Joanna Briggs12. Estes são caracterizados de forma hierárquica, dependendo do delineamento de pesquisa. Logo, a amostra final foi avaliada da seguinte forma: nível I - evidência por revisão sistemática de ensaios clínicos controlados randomizados; nível II - evidência com base em ensaio clínico controlado randomizado; nível III.1 - evidência obtida por meio de ensaios clínicos controlados, sem randomização; nível III.2 - evidência adquirida de estudos de coorte bem-delineados ou caso-controle; nível III. 3 - evidência com base em séries temporais múltiplas, com ou sem intervenção e resultados dramáticos em experimentos não controlados e, por último, nível IV - pareceres de autoridades baseados em critérios clínicos, estudos descritivos ou relatórios de comitês de especialistas 12 .

Por fim, a última etapa, consistiu na análise das variáveis analisadas: descrição das repercussões do COVID-19 para o processo de trabalho da enfermagem e o(s) elemento(s) que permitiu(ram) o novo processo de trabalho, organizadas no Quadro 2.

\section{RESULTADOS}

A partir das etapas realizadas e orientadas pelo checklist Preferred Reporting Itens for Systematic Reviews and meta-Analyses (PRISMA), a amostra final, Quadro 1 , compreendeu cinco artigos, sendo dois $(40,0 \%)$ publicado no ano 2020 e três (60,0\%) publicados em 2021.

Quanto ao delineamento metodológico foi encontrado um estudo (20,0\%) que utilizou o método desenho transversal descritivo, um $(20,0 \%)$ que utilizou estudo descritivo, um $(20,0 \%)$ ensaio reflexivo, um $(20,0 \%)$ estudo de caso e um $(20 \%)$ convergente de métodos mistos (quantitativos e qualitativos). Desta forma, os níveis de evidência correspondentes foram III e IV.

Sobre as variáveis analisadas para investigar as repercussões que a pandemia do CO-

Quadro 1 - Síntese da amostra final conforme título, objetivo, método e resultados dos artigos da amostra final. Divinópolis, MG, Brasil, 2021

\begin{tabular}{|l|l|l|l|}
\hline \multicolumn{1}{|l|}{ Título } & Objetivos & Método & Resultados \\
\hline $\begin{array}{l}\text { A case study of a collaborative } \\
\text { allied health and nursing crisis } \\
\text { response (2020)9. }\end{array}$ & $\begin{array}{l}\text { Avaliar como uma força de } \\
\text { trabalho interprofissional de uma } \\
\text { organização mais ampla poderia } \\
\text { ser apoiada para trabalhar em } \\
\text { cuidados intensivos como parte de } \\
\text { uma resposta à crise. }\end{array}$ & Estudo de caso & $\begin{array}{l}\text { A força de trabalho de enfermagem } \\
\text { respondeu às necessidades dos pacientes } \\
\text { em cuidados críticos como parte de uma } \\
\text { resposta a crises }\end{array}$ \\
\hline $\begin{array}{l}\text { An illumination of the ICN's core } \\
\text { competencies in disaster nursing } \\
\text { version 2.0: Advanced nursing } \\
\text { response to COVID-19 outbreak in } \\
\text { China (2021) }\end{array}$ & $\begin{array}{l}\text { Relatar sobre as ações e gestão } \\
\text { de incidentes dos enfermeiros de } \\
\text { prática avançada de uma equipe } \\
\text { de operação de desastres que } \\
\text { foram implantados em resposta } \\
\text { ao surto CovID-19 }\end{array}$ & Estudo descritivo & $\begin{array}{l}\text { A abordagem de resposta dos enfermei- } \\
\text { ros avançados ao covID-19 abrangeu a } \\
\text { preparação e planeamento, comunicação, } \\
\text { sistemas de gestão de incidentes, seguran- } \\
\text { ça e proteção, avaliação e intervenção }\end{array}$ \\
\hline
\end{tabular}




\begin{tabular}{|c|c|c|c|}
\hline $\begin{array}{l}\text { Trabalho do enfermeiro no con- } \\
\text { texto da pandemia de COVID-19 } \\
\text { (2021)11 }\end{array}$ & $\begin{array}{l}\text { Refletir sobre o trabalho expe- } \\
\text { rienciado pelo enfermeiro no } \\
\text { enfrentamento da pandemia de } \\
\text { COVID-19 vivenciado em um } \\
\text { hospital público do estado do Rio } \\
\text { Grande do Norte }\end{array}$ & Ensaio reflexivo & $\begin{array}{l}\text { Destacou o protagonismo eficaz da en- } \\
\text { fermagem na organização dos serviços de } \\
\text { saúde para o enfrentamento da COVID-19 e } \\
\text { o exercício da gerência dos cuidados de en- } \\
\text { fermagem em meio ao estresse, sofrimento } \\
\text { psíquico, desgastes na atuação do enfer- } \\
\text { meiro, pouco reconhecimento profissional e } \\
\text { déficits de material e pessoal nos serviços }\end{array}$ \\
\hline $\begin{array}{l}\text { Nursing care management based } \\
\text { on the Omaha system for inpa- } \\
\text { tients diagnosed with COVID-19: } \\
\text { An electronic health record study } \\
\text { (2021)12 }\end{array}$ & $\begin{array}{l}\text { Identificar as características clíni- } \\
\text { cas dos pacientes com diagnóstico } \\
\text { de COVID-19 e os problemas de } \\
\text { enfermagem existentes com base } \\
\text { no Sistema Omaha, e estabelecer } \\
\text { um plano de gerenciamento de } \\
\text { cuidados de enfermagem }\end{array}$ & Transversal descritivo & $\begin{array}{l}\text { Ao utilizar o Sistema Omaha foi possível } \\
\text { observar rapidamente os problemas dos } \\
\text { pacientes e obter melhora significativa nos } \\
\text { escores de conhecimento, comportamento } \\
\text { e status dos pacientes antes e depois da } \\
\text { intervenção permitindo diagnosticar, plane- } \\
\text { jar e implementar intervenções adequadas } \\
\text { para os pacientes COVID-19 }\end{array}$ \\
\hline $\begin{array}{l}\text { Leadership, professional quality } \\
\text { of life and moral distress during } \\
\text { COVID-19: A mixed-methods } \\
\text { approach (2021)13 }\end{array}$ & $\begin{array}{l}\text { Compreender o impacto dos } \\
\text { estressores profissionais na } \\
\text { qualidade de vida profissional e } \\
\text { sofrimento moral dos enfermeiros } \\
\text { e de outros profissionais de saúde } \\
\text { durante a pandemia COVID-19 }\end{array}$ & $\begin{array}{l}\text { Convergente de métodos } \\
\text { mistos (quantitativos e } \\
\text { qualitativos) }\end{array}$ & $\begin{array}{l}\text { A percepção de falta de apoio da liderança } \\
\text { executiva, o acesso a equipamentos de } \\
\text { proteção individual e a mudança constan- } \\
\text { te de diretrizes levaram à diminuição da } \\
\text { qualidade de vida profissional e ao aumento } \\
\text { da angústia moral }\end{array}$ \\
\hline
\end{tabular}

VID-19 trouxe para o processo de trabalho da enfermagem, os estudos apresentaram repercussões positivas, como a necessidade de os líderes de enfermagem reavaliarem a sua força de trabalho e utilizar das habilidades dos profissionais existentes para atender rapidamente à demanda de pacientes críticos pelo COVID-1913,14.

A intensificação do trabalho em equipe, o protagonismo do enfermeiro em decorrência de sua atuação eficaz e incisiva contra o novo coronavírus e a utilização do sistema Omaha para agilizar atendimentos e implementar um plano de gerenciamento também foram ações positivas evidenciadas15,16.

Porém, repercussões negativas como a redução da qualidade de vida profissional, o aumento da angústia pela falta de apoio dos líderes executivos e a necessidade de assumir funções fora de sua área regular de prática para apoiar uns aos outros no ambiente clínico também foram elencados. Estes pontos merecem atenção especial, pois emerge a reflexão sobre a reestruturação das políticas públicas de saúde, por se tratar de uma evidência que pode ser cenário de outros serviços de saúde com profissionais da enfermagem 17.

\section{DISCUSSÃO}

Em março de 2020, foi declarada a pandemia do COVID-19 e colocou em discussão a necessidade de profissionais de saúde capacitados para enfrentar uma doença

Quadro 2 - Repercussões do COVID-19 para o processo de trabalho da enfermagem. Divinópolis, MG, Brasil, 2021

\begin{tabular}{|c|c|c|}
\hline Título & $\begin{array}{l}\text { Descrição da(s) repercussão(s) do COVID-19 para o processo } \\
\text { de trabalho daenfermagem }\end{array}$ & $\begin{array}{l}\text { Elemento(s) que permitiu(ram) o novo } \\
\text { processo de trabalho da enfermagem }\end{array}$ \\
\hline $\begin{array}{l}\text { A case study of a collaborative allied he- } \\
\text { alth and nursing crisis response (2020) }\end{array}$ & $\begin{array}{l}\text { Os líderes de enfermagem precisaram rever a sua força de } \\
\text { trabalho e misturar as habilidades dos profissionais exis- } \\
\text { tentes para atender rapidamente os pacientes críticos pelo } \\
\text { COVID-19. Intensificou o trabalho em equipe }\end{array}$ & $\begin{array}{l}\text { Reforma organizacional, melhora da } \\
\text { comunicação com feedbacks mais } \\
\text { rápidos dos turnos de trabalho e } \\
\text { liderança responsiva }\end{array}$ \\
\hline
\end{tabular}




\section{artigo}

Xavier, R. V., Borges, M. S., Severino, G. S., Silva, M. D., Cardoso, J. P., Almeida, C. S., Souza,D. A. S.

Repercussões nas ações de enfermagem no cenário de pandemia do covid-19

\begin{tabular}{|l|l|l|}
\hline $\begin{array}{l}\text { An illumination of the ICN's core com- } \\
\text { petencies in disaster nursing version } \\
\text { 2.0: Advanced nursing response to } \\
\text { COVID-19 outbreak in China (2021) }\end{array}$ & Intensificou o trabalho em equipe. & $\begin{array}{l}\text { Melhora da comunicação entre geren- } \\
\text { te e equipe e entre as equipes }\end{array}$ \\
\hline $\begin{array}{l}\text { Trabalho do enfermeiro no contexto da } \\
\text { pandemia de COVID-19 (2021)11 }\end{array}$ & $\begin{array}{l}\text { Protagonismo do enfermeiro por sua atuação eficaz e incisiva } \\
\text { contra o novo coronavírus em ambiente muitas vezes com } \\
\text { falta de recursos estruturais, materiais, pessoal, desgaste físi- } \\
\text { co, emocional e baixa remuneração }\end{array}$ & $\begin{array}{l}\text { Fortalecimento dos trabalhos inter- } \\
\text { disciplinares que colaboram para a } \\
\text { pandemia da crise ocasionada pela }\end{array}$ \\
\hline $\begin{array}{l}\text { Nursing care management based on the } \\
\text { Omaha system for inpatients diagnosed } \\
\text { with COVID-19: An electronic health } \\
\text { record study (2021)12 }\end{array}$ & $\begin{array}{l}\text { Utilizou método do Sistema Omaha para agilizar atendimen- } \\
\text { tos e implementar um plano de gerenciamento. Isso aumen- } \\
\text { tou a qualidade do atendimento, pois facilitou o processo de } \\
\text { tratamento e recuperação }\end{array}$ & $\begin{array}{l}\text { Estabelecimento de diretrizes eficazes } \\
\text { para diagnosticar os problemas, pla- } \\
\text { nejar e implementar intervençães para } \\
\text { os pacientes COVID-19 }\end{array}$ \\
\hline $\begin{array}{l}\text { Leadership, professional quality of life } \\
\text { and moral distress during COVID-19: A } \\
\text { mixed-methods approach (2021)13 }\end{array}$ & $\begin{array}{l}\text { Diminuiu a qualidade de vida profissional, aumentou a angús- } \\
\text { tia entre profissionais pela falta de apoio dos líderes executi- } \\
\text { vos e por precisarem assumir funçães fora de sua área regular } \\
\text { de prática para apoiar uns aos outros no ambiente clínico }\end{array}$ & $\begin{array}{l}\text { Melhorou a colaboração inter e } \\
\text { intradisciplinar, e despertou novo } \\
\text { sentimento de respeito por outros } \\
\text { membros da equipe assistencial }\end{array}$ \\
\hline
\end{tabular}

desconhecida. A necessidade de pensar no trabalho da enfermagem frente à prevenção e o controle da infecção se fizeram necessário, visto que a sua assistência direta, contínua e prolongada com pacientes infectados configurou como o cerne dos sistemas de saúde no mundo18.

Com o crescimento dos casos, o trabalho da equipe de enfermagem se intensificou, sendo necessário que líderes de enfermagem reavaliassem a sua força de trabalho e somassem as habilidades dos profissionais para atender a alta demanda de pacientes com COVID-19. Acredita-se que essa união de habilidades e intensificação do trabalho, permitiu que os enfermeiros compartilhassem intervenções e conseguissem uma mobilização mais rápida diante dos casos, além de melhorar a comunicação da equipe e a colaboração entre si13,19.

Sabe-se, que a atividade de cuidado é de suma importância para o bem-estar da sociedade e conhecimentos específicos e de diferentes atuações profissionais são capazes de proporcionar prevenção, promoção e tratamento em saúde19. Contudo, após o início da pandemia, além das ações de cuidado com as pessoas adoecidas, houve um aumento nos procedimentos, na admissão de pacientes e na jornada de trabalho prolongada. Contudo, a partir da colaboração dos profissionais de enfermagem entre as equipes percebeu-se o fortalecimento das práticas de enfermagem tornando a assistência ágil e eficaz diante da complexidade dos quadros clínicos ocasionados pelo COVID-1914,20.

É notório que problemas como falta de insumos, menor número de profissionais na equipe, baixa remuneração e falta de valorização constituem em desafios sempre atuais para a enfermagem, e, no contexto da pandemia do COVID-19 intensificou ainda mais este problema21-23. Destaca-se que o enfermeiro por meio da Sistematização de Assistência de Enfermagem, presta não só assistência direta ao paciente, mas também lida com a gestão de ambiente, gerenciamento de pessoal, cuidado e suporte as equipes24. Para gerenciar a crise sanitária vigente, o Conselho Federal de Enfermagem (COFEN)25, elaborou diretrizes para o serviço de enfermagem, no ano de 2020. Nelas, há orientações acerca do cuidado nos casos suspeitos e confirmados de COVID-19, readequando os cuidados de enfermagem perante a crise e garantindo o máximo de segurança possível ao profissio- nal25.

Além disso, o sofrimento mental causado em decorrência da pandemia é um fator que deve ser considerado. A insegurança causada devido à falta de equipamentos de proteção individual (EPI's), o medo de se contaminar com a doença e transmitir para outras pessoas, como seus familiares e o isolamento social, são fatores que aumentam a insegurança e causam problemas mentais e, consequentemente, físicos 26 .

A pandemia também evidenciou e apresentou a população mundial, a complexidade e a necessidade dos cuidados da enfermagem para as demandas crescentes em situações de crise na saúde. Isto porque, há o senso comum de que os cuidados se restringem a higiene e medicações, logo, inúmeras pessoas aprenderam e reconheceram a ciência, a competência e todas as habilidades clínicas que integram as ações sistematizadas da enfermagem. Pôde-se perceber, também, a precariedade das estruturas direcionadas à atuação destes profissionais, visto que, $\mathrm{em}$ grande parte das instituições, faltaram insumos e EPIs, essenciais e indispensáveis para o cuidado com o paciente. Isto fez com que as equipes se desdobrassem para trabalhar com os recursos disponíveis e, mesmo as- 
sim, a enfermagem superou esse desafio ao mostrar a execução dos cuidados com qualidade e eficiência ao paciente 5 .

Somado a tudo isso, é importante destacar a exaustão física e mental desses profissionais que provocou uma crise a saúde pública, paralela a pandemia. Ao mesmo tempo em que aumentava o número de doentes hospitalizados, crescia os casos de afastamento por saúde, óbito e o alto risco de contaminação pelo COVID18. O transtorno de ansiedade generalizada, estresse crônico e a exaustão dos trabalhadores aumentaram frente à sobrecarga de trabalho27.

A pandemia trouxe ainda a perturbação do sono, irritabilidade, incapacidade de relaxar, estresse, dificuldade de concentração, tristeza, apatia e sensação negativa do futuro para os profissionais da enfermagem, além de vivenciarem diariamente debilidade do trabalho28-30.

Frente a esse cenário, embora tantos desafios tenham ameaçado a saúde, a vida $\mathrm{e}$ a integridade psicológica dos profissionais de enfermagem, destaca-se que a pandemia trouxe conhecimentos e experiências que puderam promover novas ações de cuidado para responder de forma imediata as necessidades de saúde num momento de crise sanitária mundial15,31. A pandemia também levou a enfermagem a um reconhecimento internacional, como uma profissão essencial aos cuidados à saúde. Contudo, este reconhecimento, principalmente no Brasil, não foi suficiente para levar as autoridades a oferecerem condições mais seguras de trabalho e melhorar a remuneração 32,33 .

$\mathrm{O}$ protagonismo da enfermagem durante a pandemia do COVID-19 pode ser visto em todos os âmbitos da prática de enfermagem. Um estudo evidenciou o protagonismo do enfermeiro gestor, analisando as competências gerais na prática gerencial, sendo: comunicação, tomada de decisões, liderança, administração e gerenciamento, atenção à saúde e educação permanente, logo estas ações são essenciais para a reestruturação do serviço diante da pandemia34.

O trabalho em equipe e a comunicação interprofissional também foram ressalta- das durante esta pandemia, uma vez que os sistemas de saúde se viram frente a uma demanda maior do que seus serviços poderiam oferecer e, para isso, contaram com a colaboração destes para organizar e rees-

\section{A pandemia}

trouxe ainda a

perturbação do

sono, irritabilidade,

incapacidade de

relaxar, estresse,

dificuldade de

concentração,

tristeza, apatia e

sensação negativa

do futuro para os

profissionais da

enfermagem, além

de vivenciarem

diariamente

\section{debilidade do}

trabalho

truturar a assistência à saúde de forma mais segura e eficiente. Além de promoverem a capacitação de novos contratados e dos profissionais já atuantes, também foi essencial que o vínculo e comunicação entre eles estivessem alinhados para que não houvesse divergências nas informações passadas à po- pulação35.

De acordo com a literatura, o uso de tecnologias e protocolos durante a pandemia aumentou trazendo uma resposta imediata e mais eficaz para as situações de crise na saúde. Isso se confirma no estudo de Souza et al.26, que afirma a importância da elaboração de novos protocolos que embasaram a atuação da Enfermagem, fundamentando-as em evidências científicas, esclarecendo dúvidas e consolidando o conhecimento da equipe. No que diz respeito às tecnologias, Duarte et al.5 afirmam que estas colaboraram diretamente para o combate da pandemia, seja no processo de teleconsulta de enfermagem, ou até mesmo na divulgação de informações sobre prevenção, cuidados, orientações, esclarecimentos, dentre outros.

Esta pesquisa apresenta como limitações o fato de considerar os resultados de publicações num período delimitado, ou seja, não inclui estudos publicados posteriormente que poderiam trazer novos desdobramentos para a investigação. As reflexões e as observações abrem a perspectiva para investimentos em pesquisas na enfermagem para melhorar os protocolos de atendimento clínico nas situações de urgências, pandemias e crises sanitárias locais. Além disso, destaca sobre a importância de futuros trabalhos investigarem como o trabalho de enfermagem se diferencia em diferentes estados, regiões e países para caracterizar as demandas desses profissionais e melhorar as condições de trabalho, remuneração $e$ aumentar sua segurança física e emocional.

\section{CONCLUSÕES}

As repercussões das ações de enfermagem no cenário de pandemia do $\mathrm{CO}$ VID-19 permitiram levantar discussões para implementar, reestruturar e oferecer melhores condições de trabalho, saúde e bem-estar aos trabalhadores de enfermagem. Além destes, a remuneração, apoio emocional, qualificação continuada, investimento em tecnologias e pesquisas são elementos necessários para minimizar os impactos negativos de uma pandemia, bem como qualquer outra situação de crise na saúde. 


\section{artigo}

Xavier, R. V., Borges, M. S., Severino, G. S., Silva, M. D., Cardoso, J. P., Almeida, C. S., Souza,D. A. S.

Repercussões nas ações de enfermagem no cenário de pandemia do covid-19

\section{REFERÊNCIAS}

1 Medina MG, Giovanella L, Bousquat A, Mendonça MEM, Aquino R. Primary healthcare in times of COVID-19: what to do? Cad. Saúde Pública [Internet]. 2020 [cited 2021 nov19];36(8):e00149720. Available from: http://cadernos.ensp. fiocruz.br/static/arquivo/1678-4464-csp-36-08-e00149720. pdf.

2 World Health Organization (WHO). State of the World's Nursing 2020. Executive Sumary: Investing in Education, Jon and Leadership. Geneva: WHO, 2020.

3 Silva MCN, Cunha CLF, Ximenes Neto FRG, Santos JLG, Lourenção LG, Freire MP, et al. Protagonismo da Enfermagem Brasileira no combate à COVID-19. Enferm Foco. 2020;11(1):8-9.

4 Freire NP, Castro DA, Fagundes MCM, Ximenes Neto FRG, Cunha ICKO, Silva MCN. Notícias sobre a Enfermagem Brasileira na pandemia da COVID-19. Acta Paul Enferm [Internet]. 2021 [citado 19 nov 2021];34:eAPE02273. Disponivel em: https://doi. org/10.37689/acta-ape/2021A002273.

5 Barros ALBL de, Silva VM da, Santana RF, Cavalcante AMRZ, Vitor AF, Lucena $A F$, et al. Contribuições da rede de pesquisa em processo de enfermagem para assistência na pandemia de COVID-19. Rev Bras Enferm [Internet]. 2020 [citado 09 dez 2021];73(suppl 2):e20200798. Disponivel em: https://doi. org/10.1590/0034-7167-2020-0798.

6 Silva DKM da, Santos FA dos, Duarte RB, Batista e Silva DP, Soares DG, Cabral RL. Os desafios da enfermagem como coordenadora do cuidado frente à pandemia da COVID-19. Saúde Colet [Internet]. 2021 [citado 09 dez 2021];11(65):5896905. Disponivel em: https://doi.org/10.36489/saudecoletiva.2021v11i65p5896-5905.

7 Reis LM dos, Lago PN do, Carvalho AHS, Nobre VNN, Guimarães APR. Atuação da enfermagem no cenário da pandemia COVID-19. Nursing [Internet]. 2020 [citado 09 dez 2021];23(269):476568. Disponivel em: https://doi.org/10.36489/nursing .2020v23i269p4765-4772.

8 Carvalho MRS, Gomes NP, Corrêa RM, Oliveira MAS, Lima NS, Santos JK dos. Sofrimento psíquico em profissionais de saúde: uma reflexão em tempos da pandemia pela COVID-19. Saúde Colet [Internet]. 2021 [citado 09 dez 2021];11(COVID):7019-32. Disponivel em: http://revistas.mpmcomunicacao.com.br/index. php/saudecoletiva/article/view/1758.

9 Duarte MLC, Silva DG, Bagatini MMC. Enfermagem e saúde mental: uma reflexão em meio à pandemia de coronavírus. Rev Gaúcha Enferm [Internet]. 2020 [citado 19 nov 2021];42(esp):e20200140. Disponivel em: https://www.scielo.br/j/rgenf/a/ MnRHwqvgq3kTrHQ3JPSLR7H/?format=pdf\&lang=pt

10 Hopia H, Latvala E, Liimatainen L. Reviewing the methodology of an integrative review. Scand J Caring Sci. 2016;30(4):662-9.

11 Galvão TF, Pansani TSA, Harrad D. Principais itens para relatar Revisões sistemáticas e Meta-análises: A recomendação PRISMA. Epidemiol Serviç Saúde. 2015;24(2):335-42.

12 Peters MDJ, Godfrey C, Mclnerney P, Baldini Soares C, khalil H,
Parker D. Chapter 11: Scoping Reviews. In: Aromataris E, Munn Z (Editors). Joanna Briggs Institute Reviewer's Manual. The Joanna Briggs Institute, 2017.

13 Hales P, White A, Eden A, Hurst R, Moore S, Riotto C, et al. A case study of a collaborative allied health and nursing crisis response. J Interprof Care. 2020;34(5):614-21.

14 Mao X, Yang Q, Li X, Chen X, Guo C, Wen X, et al. An illumination of the ICN's core competencies in disaster nursing version 2.0: Advanced nursing response to COVID-19 outbreak in China. J Nurs Manag 2021;29(3):412-20.

15 Silva VGF da, Silva BN da, Pinto ESG, Menezes RMP de. Trabalho do Enfermeiro no contexto da pandemia de COVID-19. Rev Bras Enferm [Internet]. 2021 [citado 19 nov 2021];74(Suppl 1):e20200594.Disponivelem:https://doi.org/10.1590/00347167-2020-0594

16 Ardic A, Turan E. Nursing care management based on the Omaha system for inpatients diagnosed with COVID-19: An electronic health record study. J Adv Nurs 2021;77(6): 2709-17.

17 Ness MM, Saylor J, DiFusco LA, Evans K. Leadership, professional quality of life and moral distress during COVID-19: A mixed-methods approach. J Nurs Manag 2021;29(8):2412-22.

18 Miranda FMA, Santana L de L, Pizzolato AC, Saquis LMM. Condições de trabalho e o impacto na saúde dos profissionais de enfermagem frente a Covid-19. Cogitare Enferm [Internet]. 2020 [citado 09 nov 2021];25:e72702. Disponivel em: http://dx. doi.org/10.5380/ce.v25i0.72702

19 Gandra EC, Silva KL, Passos HR, Schreck RSC. Enfermagem brasileira e a pandemia de COVID-19: desigualdades em evidência. Esc Anna Nery [Internet]. 2021 [citado 09 nov 2021];25(spe):e20210058. Disponivel em: https://doi. org/10.1590/2177-9465-EAN-2021-0058.

20 Vedovato TG, Andrade CB, Santos DL, Bitencourt AM, Almeida LP, Sampaio FF. Trabalhadores(as) da saúde e a COVID-19: condições de trabalho à deriva? Rev Bras Saude Ocup [Internet]. 2021 [citado 19 nov 2021];46:e1. Disponível em:https://doi. org/10.1590/2317-6369000028520

21 Gallasch CH, Cunha ML, Pereira LAS, Silva-Junior JS. Prevenção relacionada à exposição ocupacional do profissional de saúde no cenário da COVID-19. Rev Enferm UERJ [internet]. 2020 [citado 19 nov 2021];28:e49596. Disponivel em: https:// www.e-publicacoes.uerj.br/index.php/enfermagemuerj/article/ view/49596/33146

22 Pérez-Júnior EF, David HMSL. Trabalho de enfermagem e precarização: uma revisão integrativa. Enferm Foco. 2018;9(4):7176.

23 Filho JMJ, Assunção AA, Algranti E, Garcia EG, Saito CA, Maeno M. A saúde do trabalhador e o enfrentamento da COVID-19. Rev Bras Saúde Ocup [Internet]. 2020 [citado 19 nov 2021];45:e14. Disponivel em: https://www.scielo.br/j/rbso/a/Km3dDZSWmGgpgYbjgc57RCn/?format=pdf\&lang=pt 


\section{REFERÊNCIAS}

24 Bitencourt JVOV, Meschial WC, Frizon G, Biffi P, Souza JB de, Maestri $E$. Nurse's protagonism in structuring and managing a specific unit for covid-19. Texto e Contexto Enferm [Internet]. 2020 [cited 2021 nov 19];29:e20200213. Available from: https:// pesquisa.bvsalud.org/portal/resource/pt/biblio-1127494.

25 Conselho Federal de Enfermagem (COFEN). Cofen publica diretrizes para serviços de Enfermagem frente o COVID-19 [Internet]. [citado 09 nov 2021]. Disponivel em:http://www.cofen. gov.br/cofen-publica-diretrizes-para-servicos-de-enfermagem-frente-o-covid-19_78031.html.

26 Souza NVDO, Carvalho EC, Soares SSS, Varella TCMML, Pereira SRM, Andrade KBS. Nursing work in the COVID-19 pandemic and repercussions for workers' mental healyh. Rev Gaúcha Enferm [Internet]. 2021 [cited 2021 nov 19];42(spe):e20200225. Available from: https://doi.org/10.1590/1983-1447.2021.20200225.

27 Teixeira CFS, Soares CM, Souza EA, Lisboa ES, Matos IC, Andrade $L R$, et al. A saúde dos profissionais de saúde no enfrentamento da pandemia de Covid-19. Ciênc Saúde Coletiva. 2020;25(9):3465-74.

28 Ramos-Toescher, Aline Marcelino et al. Saúde mental de profissionais de enfermagem durante a pandemia de COVID-19: recursos de apoio. Esc Anna Nery [Internet]. 2020 [citado 19 nov 2021];24(spe):e20200276. Disponivel em: https://doi. org/10.1590/2177-9465-EAN-2020-0276.

29 Leonel F. Pesquisa analisa o impacto da pandemia entre profissionais de saúde. Fundação Oswaldo Cruz. Disponível em: https://portal.fiocruz.br/noticia/pesquisa-analisa-o-impacto-da-pandemia-entre-profissionais-de-saude

30 Quadros A de, Fernandes MTC, Araújo BR, Caregnato RCA. Desafios da Enfermagem Brasileira no Combate da COVID-19: uma reflexão. Enferm Foco 2020;11(1 esp):78-83.

31 International Concil of Nurses (ICN). Nurses a voice to lead: a vision for future healthcare. Geneva: ICN; 2021.

32 Conselho Regional de Enfermagem de Rondônia (COREn RO). $90 \%$ dos Conselhos de Enfermagem do mundo veem risco de aumento no abandono da profissão. Rondônia: COREn; 2021.

33 Nishiyama JAP, Moraes RMR, Magalhães AMM de, Nicola $A L$, Trevilato DD, Oliveira JLC de. Dimensões laborais, éticas e políticas do dimensionamento de pessoal de enfermagem diante da COVID-19. Esc Anna Nery [Internet]. 2020 [citado 19 nov 2021];24(spe):e20200382.Disponivel em: https://doi. org/10.1590/2177-9465-EAN-2020-0382.

34 Treccossi SPC, Ferreira JC, Oliveira RM, Santos RP, Carvalho ARS. Protagonismo da enfermagem na organização de uma unidade para assistência à pacientes com Coronavírus. J Nurs Health [Internet]. 2020 [citado 19 nov 2021];10(n. esp.):e20104039. Disponivel em: https://periodicos.ufpel.edu.br/ojs2/index.php/ enfermagem/article/view/19859/12100

35 Gerolin FS, Pires AM, Nascimento C, Schimitt C, Bucione FTS, Rocha JSA, et al. Ações de lideranças da Enfermagem na organização do atendimento hospitalar a pacientes com COVID-19. Enferm Foco. 2020;11(2.esp):207-11. 